\title{
Analysis of Measurement Uncertainty in THz-TDS
}

\author{
W. Withayachumnankul, H. Lin, S. P. Mickan, B. M. Fischer, and D. Abbott \\ Centre for Biomedical Engineering and School of Electrical \& Electronic Engineering, \\ The University of Adelaide Adelaide, SA 5005, Australia
}

\begin{abstract}
Measurement precision is often required in the process of material parameter extraction. This fact is applicable to terahertz time-domain spectroscopy (THz-TDS), which is able to determine the optical/dielectric constants of material in the T-ray regime. Essentially, an ultrafast-pulsed THz-TDS system is composed of several mechanical, optical, and electronic parts, each of which is limited in precision. In operation, the uncertainties of these parts, along with the uncertainties introduced during the parameter extraction process, contribute to the overall uncertainty appearing at the output, i.e. the uncertainty in the extracted optical constants. This paper analyzes the sources of uncertainty and models error propagation through the process.
\end{abstract}

Keywords: THz-TDS, T-rays, spectroscopy, measurement uncertainty, optical constants, noise, ultrafast laser systems

\section{INTRODUCTION}

T-rays are electromagnetic radiation in the frequency range between 0.1 and $10 \mathrm{THz}$. This frequency range is higher than the microwave but lower than infrared, being at the border between the electronic and optic worlds. In the past, this so-called terahertz gap was difficult to access because of poor hardware capability, high atmospheric absorption, and strong thermal background noise.

Terahertz time-domain spectroscopy (THz-TDS) represents a breakthrough in T-ray generation and detection. At the generation end, it most commonly employs photoconductive antenna (PCA $)^{1}$ or nonlinear electro-optic (EO) crystal $^{2}$ to convert an ultrashort optical burst into a coherent T-ray pulse. At the detection end, an optically gated antenna or EO crystal is employed, thus enabling the recording of high-SNR time-resolved T-ray waveforms.

A T-ray waveform transmitted through a material sample is rich in information, since its amplitude and phase are linearly altered by the material's response. Sample and reference waveforms, once converted by a fast-Fourier transform into the frequency domain, can be processed to extract the frequency-dependent optical constants and related quantities of materials. ${ }^{3,4}$

Despite the high SNR of T-ray signals, several sources of fluctuations and variations, involved in the signal generation and detection of THz-TDS, can affect the reliability of the extracted optical constants. These fluctuations are, for instance, ultrafast-laser instability, ${ }^{5,6}$ water-vapor-induced fluctuations, ${ }^{7}$ reflections, ${ }^{8}$ blackbody radiation, optical and electronic noise, ${ }^{9,10}$ etc. In general, all of these sources of fluctuations are undesired in a T-ray signal, and their effects are removed or reduced by some means.

But more specifically, the uncertainty being investigated in this work refers to only fluctuations or variations that are not reproducible. Any fluctuation that is reproducible over several measurements, including watervapor-induced fluctuations and reflections after a main pulse, does not increase the system's uncertainty, and thus is not considered here.

In this paper, we show how the sources of uncertainty propagate to the output, and we derive the mathematical relation between each source variance and the output variance. In addition to the uncertainty arising from the

Email addresses: withawat@eleceng.adelaide.edu.au (W. Withayachumnankul); hlin@eleceng.adelaide.edu.au (H. Lin); spmickan@eleceng.adelaude.edu.au (S. P. Mickan); bfischer@eleceng.adelaide.edu.au (B. M. Fischer); dabbott@eleceng.adelaide.edu.au (D. Abbott)

Photonic Materials, Devices, and Applications II, edited by Ali Serpengüzel,Gonçal Badenes, Giancarlo Righini Proc. of SPIE Vol. 6593, 659326, (2007) · 0277-786X/07/\$18 - doi: 10.1117/12.721876 
hardware, the analysis also considers sources of uncertainty that might take place throughout the parameter extraction process. The proposed analysis is applicable to either PCA or EO generation and detection systems.

The paper is organized as follows: Section 2 presents background on the THz-TDS system and parameter extraction process, which underlies the theory of the system uncertainty; Noise and uncertainties in THz-TDS are reviewed in Section 3; Section 4 provides a means to determine the variance and covariance of a general function; An analysis of uncertainty from various sources, propagating down the THz-TDS measurement and parameter extraction process, is given in Section 5; Based on the analysis in previous section, Section 6 considers the effects of each uncertainty source independently; The paper ends with Section 7, discussion and conclusion.

\section{THZ-TDS AND MATERIAL PARAMETER EXTRACTION}

\subsection{THz-TDS system}

The THz-TDS system shown in Figure 1 is mainly composed of an ultrafast optical laser, T-ray emitter/receiver, an optical delay line, a set of mirrors, and a material sample. The ultrafast optical pulse is divided into two paths, a probe beam and a pump beam, by a beam splitter. At the emitter, the optical pump beam stimulates T-ray pulsed radiation via either charge transport ${ }^{1}$ or optical rectification effect, ${ }^{2}$ depending on the emitter type. The diverging T-ray beam is collimated and focuses onto the sample by a lenses and a pair of parabolic mirrors. After passing through the sample, the T-ray beam is re-collimated and focused onto the receiver by an identical set of lenses and mirrors. At the receiver, the initially divided probe beam optically gates the T-ray receiver with a short time duration compared with the arriving T-ray pulse duration. Synchronizing between the optical gating pulse and the T-ray pulse allows the coherent detection of the T-ray signal at a time instance. A complete temporal scan of the T-ray signal is enabled by the discrete micro-motion of a mechanical stage controlling the optical delay line.

\subsection{Parameter extraction process}

The aim of THz-TDS is to find the frequency-dependent optical or dielectric constants of materials. However, the signals available from THz-TDS are, as the name implies, based in the time domain, and has geometric

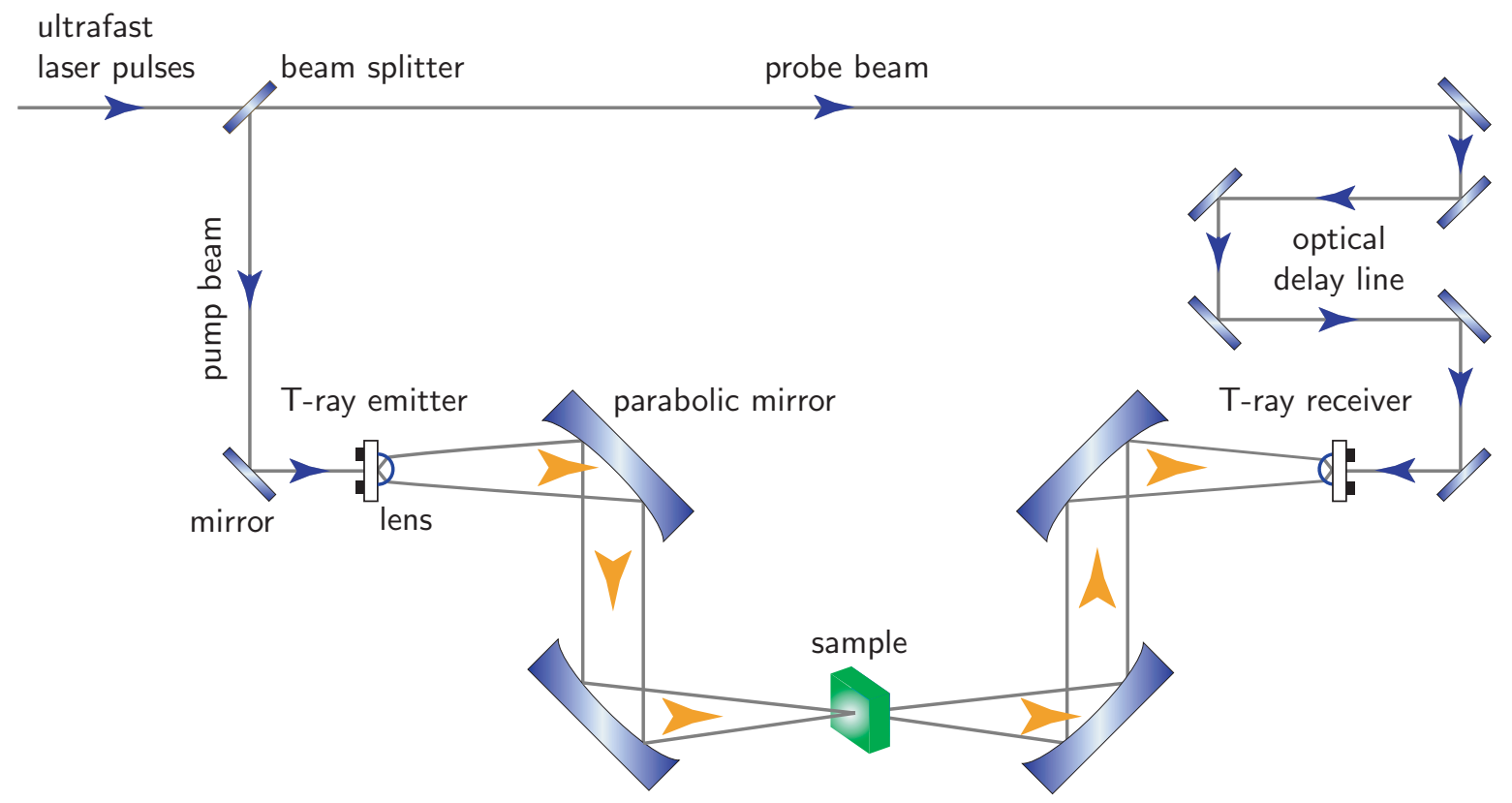

Figure 1. THz-TDS system configured in transmission mode with PCA generation and detection. The system consists of an ultrafast optical laser, T-ray emitter/receiver, an optical delay line, a set of mirrors, and a material sample. The optical beam paths are indicated by small blue arrowheads, and the T-ray beam paths by the large orange arrowheads. 
implications, i.e. reflection and refraction. This necessitates a computational means to extract the constants from such signals. In brief, the material parameter extraction of THz-TDS requires the measurement of two signals, one for the sample material and the other as a reference (which is the signal when the sample is removed). Both signals are Fourier-transformed and deconvolved with each other, yielding the complex transfer function of a material in the frequency domain. The optical or dielectric constants are then extractable from the transfer function based on the geometrical analysis of wave propagation and the knowledge of sample's orientation, thickness, and the refractive index of air.

\subsection{Assumptions on the experiment}

The assumptions of the THz-TDS measurement considered here are that (i) a sample under measurement is a dielectric slab with parallel, flat surfaces, (ii) scattering and reflections at the surfaces are negligible, (iii) the incident angle of the T-ray beam is normal to the sample surface, and (iv) the reference signal is measured under the same conditions except for the absence of the sample.

\section{NOISE AND UNCERTAINTIES IN THZ-TDS}

Previous work on THz-TDS intensively discussed noise in the system, which can be divided into three sub-topics: noise identification, noise reduction, and the effects of noise on THz-TDS characteristics. Here, we briefly cover the literature on these topics:

\subsection{Noise-source identification}

In 1990, van Exter and Grischkowsky ${ }^{9}$ characterized noise sources in a PCA receiver. The major noise sources include: (i) thermal Johnson-Nyquist noise generated by charge carriers in a substrate. It plays a role in the absence of the T-ray incident electrical field, whether with or without optical gating pulses. (ii) thermal background noise inducing a random voltage across the antenna. Due to incoherent thermal radiation and the integrating scheme of detector, the thermal noise is significantly reduced. Other relevant noise sources are quantum fluctuations and laser \& shot noise.

Duvillaret et al..$^{10}$ highlighted on the origins of noise and the uncertainties in the optical constants arising from noise. In that paper, forward and backward models were developed as functions of measured pulses. The forward model predicts the error in optical constants from noise plaguing the reference and sample spectra, whereas the backward model predicts characteristics of three different noise sources from the reference and sample spectra. It was found that the emitter noise, linked to the laser fluctuation, dominates all other noise contributions for transparent materials. On the other hand, the detector noise or noise floor affects the signals for absorbing materials

\subsection{Noise reduction}

Duvillaret et al., ${ }^{11}$ similar to Jepsen et al, ${ }^{12}$ developed an analytical model of a T-ray pulse generated from and detected by photoconductive antennas. The model shows that the detected pulse dynamics depend on the laser average power, laser pulse duration, carrier collision time, carrier recombination time, DC bias, and are strongly influenced by the carrier lifetime and the laser pulse duration. The model, together with a previous finding on noise characteristics, ${ }^{10}$ enables optimization of these parameters to gain the best measurement performance. It was found that the laser pulse duration should be as short as possible, whereas the carrier lifetime should be in accordance with the frequency range of interest.

In order to cope with the laser fluctuation, T-ray differential time-domain spectroscopy (DTDS) proposed by Lee et al. ${ }^{13}$ and Jiang et al. ${ }^{14}$ measures the difference between sample and reference waveforms by moving the sample in and out of the T-ray beam at a considerable rate. The difference effectively cancels out the effects of slow-varying laser fluctuation, enable sensing and characterizing thin films with the thickness in the order of submicron range.

Noise in a T-ray signal could be removed by a digital signal processing technique such as wavelet denoising. Ferguson and Abbott ${ }^{15,16}$ tested a wavelet denoising technique on noisy T-ray signals. Soft wavelet denoising is shown to improve the SNR of signals, particularly when signals are strongly absorbed by biological sample, 
and the Coiflet order 4 wavelet is found to be optimal with up to $10 \mathrm{~dB}$ in noise reduction. Nevertheless, the optimal wavelet is dependent upon the T-ray pulse shape, and wavelet filtering could introduce artifacts. A more appealing DSP approach is Spatially Variant Moving Average Filter (SVMAF) proposed by Pupeza et al. ${ }^{17}$ The SVMAF algorithm establishes the confidence interval of a transfer function via the measurement uncertainty. The frequency-dependent material parameters extracted from the averaged transfer function are smoothed out over the frequency range. The new smoothed value at any frequency is accepted, if it constructs a new transfer function value that is confined within the confidence interval, and is rejected otherwise.

\subsection{Effects of noise on THz-TDS characteristics}

Ultimately, the noise in a THz-TDS system limits the spectral resolution. Xu et al. ${ }^{18}$ and Mickan et al. ${ }^{19}$ proved that the highest spectral resolution at a particular frequency range depends on the maximum time duration, which depends on the SNR at that frequency. In order to achieve a higher frequency resolution, it is necessary to increase the dynamic range of the system either by increase transmitted energy or decreasing the noise floor.

Jepsen and Fischer ${ }^{20}$ established the relation between the dynamic range of the detectable absorption coefficient and the system characteristics for transmission and reflection THz-TDS. It is found that, for transmission mode THz-TDS, the maximum measurable range of the absorption coefficient is limited by the dynamic range of T-ray signal. On the other hand, for reflection mode THz-TDS, the dynamic range is only limited by scan-to-scan reproducibility of the signal.

It would appear that the previous literature addressed the noise issues in THz-TDS comprehensively. However, a closely related subject, the uncertainty of the system, is left unexplored. Based on the previous work on noise, an uncertainty analysis for THz-TDS is established in this paper.

\section{VARIANCE AND COVARIANCE OF FUNCTION}

For a deterministic system, an output parameter, $y$, can be expressed or, at least, approximated as a function of input parameters $x_{1}, x_{2}, \ldots$, or

$$
y=f\left(x_{1}, x_{2}, \ldots\right) .
$$

If the uncertainty appears at the input parameters as a result of measurement, it will propagate to the output through this function. Given that the uncertainty has the normal distribution, the output mean is related to the input means via

$$
\bar{y}=f\left(\bar{x}_{1}, \bar{x}_{2}, \ldots\right)
$$

\subsection{Variance of function}

The output, $y_{i}$, that is caused by the uncertainty of the input, $x_{1 i}-\bar{x}_{1}, x_{2 i}-\bar{x}_{2}, \ldots$, can be approximated by Taylor series to the first order,

$$
\begin{aligned}
y_{i} & \approx \bar{y}+\left(x_{1 i}-\bar{x}_{1}\right) \frac{\partial f\left(\bar{x}_{1}\right)}{\partial x_{1}}+\left(x_{2 i}-\bar{x}_{2}\right) \frac{\partial f\left(\bar{x}_{2}\right)}{\partial x_{2}}+\ldots, \quad \text { or } \\
y_{i}-\bar{y} & \approx\left(x_{1 i}-\bar{x}_{1}\right) \frac{\partial f\left(\bar{x}_{1}\right)}{\partial x_{1}}+\left(x_{2 i}-\bar{x}_{2}\right) \frac{\partial f\left(\bar{x}_{2}\right)}{\partial x_{2}}+\ldots .
\end{aligned}
$$

Note that to keep the notation concise, from now on, $\partial f(\bar{x}) / \partial x$ will be represented by $\partial f / \partial x$. Squaring and weighted summing over $M$ measurements gives

$$
\begin{aligned}
\frac{1}{M} \sum_{i=1}^{M}\left(y_{i}-\bar{y}\right)^{2} \approx & \frac{1}{M} \sum_{i=1}^{M}\left[\left(x_{1 i}-\bar{x}_{1}\right) \frac{\partial f}{\partial x_{1}}+\left(x_{2 i}-\bar{x}_{2}\right) \frac{\partial f}{\partial x_{2}}+\ldots\right]^{2} \\
= & \frac{1}{M} \sum_{i=1}^{M}\left(x_{1 i}-\bar{x}_{1}\right)^{2}\left(\frac{\partial f}{\partial x_{1}}\right)^{2}+\frac{1}{M} \sum_{i=1}^{M}\left(x_{2 i}-\bar{x}_{2}\right)^{2}\left(\frac{\partial f}{\partial x_{2}}\right)^{2}+ \\
& \frac{2}{M} \sum_{i=1}^{N}\left(x_{1 i}-\bar{x}_{1}\right)\left(x_{2 i}-\bar{x}_{2}\right) \frac{\partial f}{\partial x_{1}} \frac{\partial f}{\partial x_{2}}+\ldots
\end{aligned}
$$


The above equation can be written in terms of variance,

$$
\sigma_{y}^{2}=\sigma_{x_{1}}^{2}\left(\frac{\partial f}{\partial x_{1}}\right)^{2}+\sigma_{x_{2}}^{2}\left(\frac{\partial f}{\partial x_{2}}\right)^{2}+2 \sigma_{x_{1} x_{2}}^{2} \frac{\partial f}{\partial x_{1}} \frac{\partial f}{\partial x_{2}}+\ldots
$$

If the input parameters are independent from one another, the above equation is simplified to

$$
\sigma_{y}^{2}=\sigma_{x_{1}}^{2}\left(\frac{\partial f}{\partial x_{1}}\right)^{2}+\sigma_{x_{2}}^{2}\left(\frac{\partial f}{\partial x_{2}}\right)^{2}+\ldots
$$

\subsection{Covariance of function}

Provided that there is a function $g$, sharing the same set of input parameters as function $f$, or $y=f\left(x_{1}, x_{2}, \ldots\right)$ and $z=g\left(x_{1}, x_{2}, \ldots\right)$, the series expansion for the function $g$, analogous to Equation 3, is

$$
z_{i}-\bar{z} \approx\left(x_{1 i}-\bar{x}_{1}\right) \frac{\partial g}{\partial x_{1}}+\left(x_{2 i}-\bar{x}_{2}\right) \frac{\partial g}{\partial x_{2}}+\ldots
$$

Multiplying Equation 7 with Equation 3 yields

$$
\begin{aligned}
\left(y_{i}-\bar{y}\right)\left(z_{i}-\bar{z}\right)= & \left(x_{1 i}-\bar{x}_{1}\right)^{2} \frac{\partial f}{\partial x_{1}} \frac{\partial g}{\partial x_{1}}+\left(x_{2 i}-\bar{x}_{2}\right)^{2} \frac{\partial f}{\partial x_{2}} \frac{\partial g}{\partial x_{2}}+\ldots+ \\
& \left(x_{1 i}-\bar{x}_{1}\right)\left(x_{2 i}-\bar{x}_{2}\right)\left(\frac{\partial f}{\partial x_{1}} \frac{\partial g}{\partial x_{2}}+\frac{\partial f}{\partial x_{2}} \frac{\partial g}{\partial x_{1}}\right)+\ldots
\end{aligned}
$$

By weighted summing over $M$ measurements, and assuming the independence of input parameters, the covariance is

$$
\sigma_{y z}^{2}=\sigma_{x_{1}}^{2} \frac{\partial f}{\partial x_{1}} \frac{\partial g}{\partial x_{1}}+\sigma_{x_{2}}^{2} \frac{\partial f}{\partial x_{2}} \frac{\partial g}{\partial x_{2}} \ldots
$$

\section{UNCERTAINTY PROPAGATION}

Propagation of uncertainties during the THz-TDS measurement and the parameter extraction process is shown in Figure 2. The earliest uncertainty involves error in positioning of the optical delay line stage, which mechanically delays the optical probe pulse (or equivalently the pumping pulse) by using a pair of moving mirrors. This uncertainty affects the sampling time of an optically-gated detector. The uncertainty in the sampling time, combined with electronic and optical noise, gives rise to uncertainty in the sampled T-ray pulse amplitude.

When measured T-ray signals, including reference and sample signals, are pre-processed to extract the optical constants, the amplitude error propagates through the Fourier transform and deconvolution stages. An additional uncertainty occurs during the process of phase unwrapping.

The parameter extraction process requires knowledge of the sample thickness and its orientation. This step introduces uncertainty in the thickness measurement and uncertainty in the sample alignment. In addition, the error in air refractive index estimation gives rise to the overall uncertainty. Eventually, all these uncertainties accumulate and contribute to the uncertainty in the extracted optical constants.

The following subsections develop mathematical models representing uncertainty connection at each stage of measurement.

\subsection{Optically-gated sampling}

The deviation of the delay line position from the correct position, $\Delta x$, results in the deviation in the sampling time, $\Delta t$, of an electro-optic or photoconductive sampling system. This situation is illustrated in Figure 3. Since the optical probe beam travels in free space, a simple relation between the deviation in the delay line position and the change in the sampling time for a normal delay stage can be established as

$$
\Delta t=2 \Delta x / c .
$$


The multiplication by two indicates that the optical path is twice that of the deviation length $\Delta x$ (see Figure 3a). The variance of the sampling time, $\sigma_{t}^{2}$, is then

$$
\sigma_{t}^{2}=\frac{4 \sigma_{x}^{2}}{c^{2}}
$$

where $\sigma_{x}^{2}$ is the uncertainty in delay line position.

\subsection{Amplitude detection}

The amplitude of a temporal waveform measured by an optically-gated sampling system is influenced by change in the sampling time. Through the first order expansion of the Taylor series, the measured amplitude assigned to a time index $k$ is a function of the amplitude at a correct time $t_{k}$ plus the amplitude derivative multiplied by the sampling-time deviation (see Figure 3b), or

$$
E(k)=E\left(t_{k}\right)+\Delta t \frac{d E\left(t_{k}\right)}{d t},
$$

where $t_{k} \equiv k \tau$ and $\tau$ is the sampling interval. The variances of $E\left(t_{k}\right)$ and $\Delta t$ yield the variance of the measured amplitude of ${ }^{21}$

$$
\sigma_{E}^{2}(k)=\sigma_{E}^{2}\left(t_{k}\right)+\sigma_{t}^{2}\left[\frac{\partial E\left(t_{k}\right)}{\partial t}\right]^{2}
$$

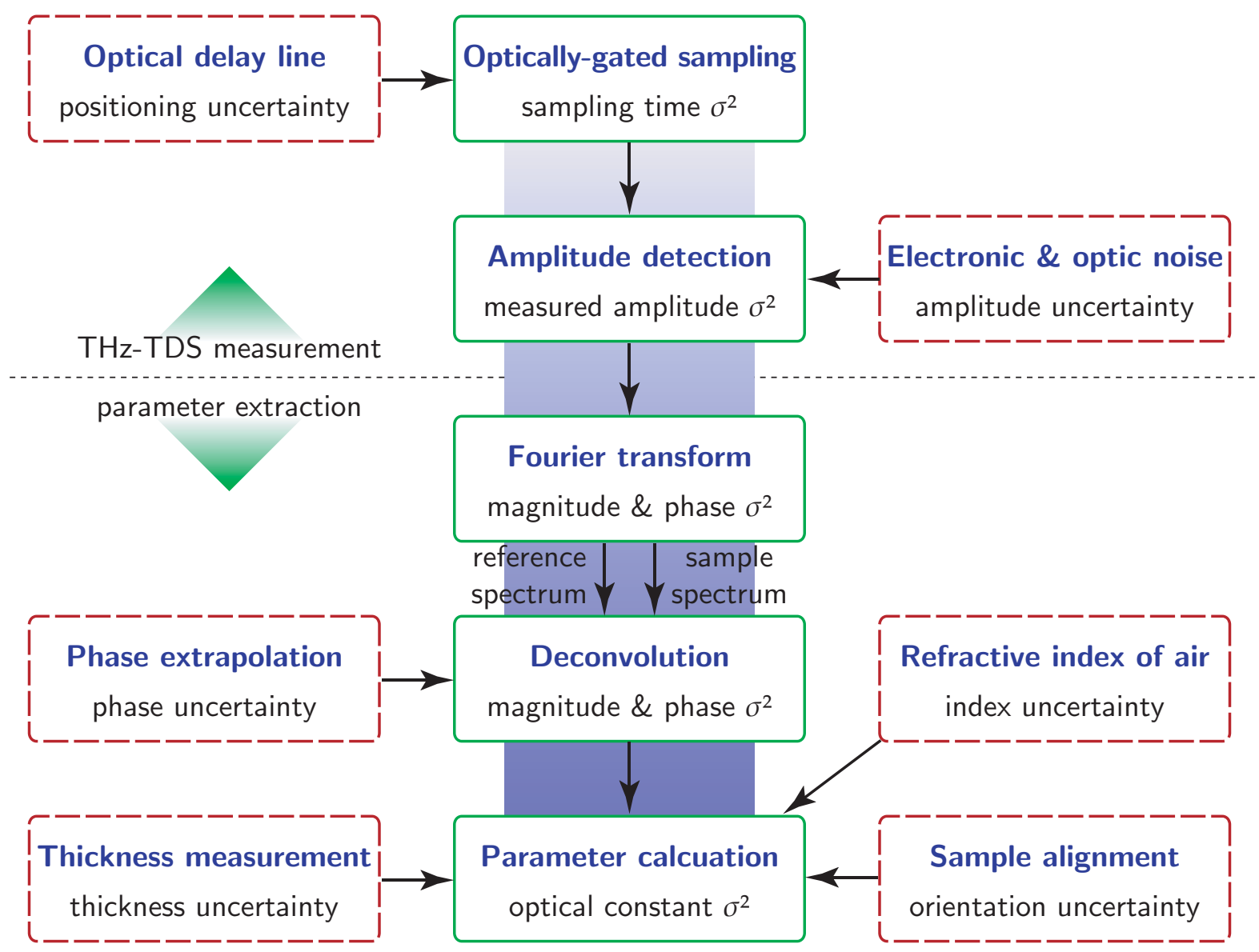

Figure 2. Propagation of uncertainties. The uncertainty sources (red dashed lines) can take place in both the THz-TDS measurement and the parameter extraction process. They cause the variance, $\sigma^{2}$, propagating down the process, and eventually contribute to the variance in the extracted optical constants. 


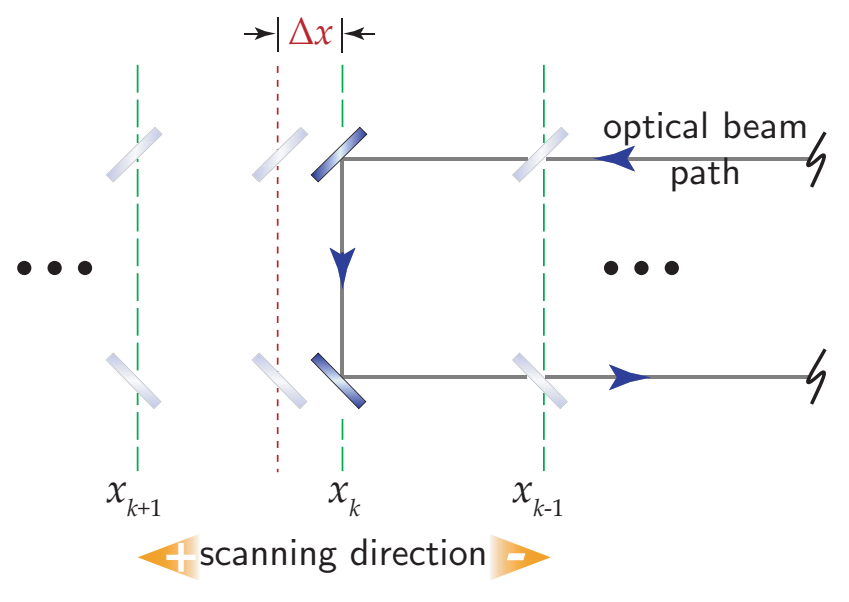

(a) mechanical delay stage for optical beam

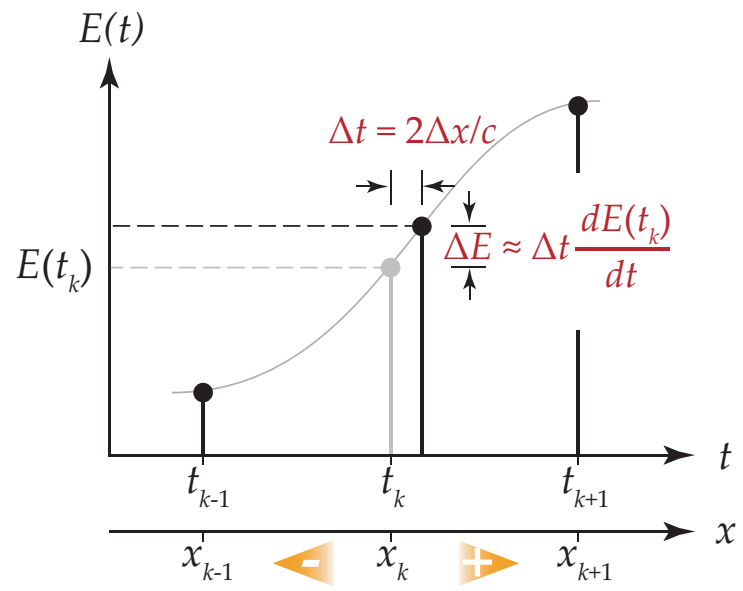

(b) corresponding sampled time-domain signal

Figure 3. Delay line stage and a sampled signal. In Figure (a), the normal positions of a pair of mirrors are $\left\{\ldots, x_{k-1}, x_{k}, x_{k+1}, \ldots\right\}$ as marked by the green dash lines. These sampling positions correspond to sampling times of $\left\{\ldots, t_{k-1}, t_{k}, t_{k+1}, \ldots\right\}$, as shown in Figure (b). The error in positioning a pair of mirrors by distance $\Delta x$ (red dotted line) at $x_{k}$ leads to the error in sampling time $\Delta t$ and ultimately to the error in amplitude measurement $\Delta E$. Note that the figure excludes the effect of noise.

The amplitude variance $\sigma_{E}^{2}\left(t_{k}\right)$ is caused by noise, which is amplitude-dependent, ${ }^{10}$ and can be expressed by a polynomial series:

$$
\sigma_{E}^{2}\left(t_{k}\right)=A E^{2}\left(t_{k}\right)+B\left|E\left(t_{k}\right)\right|+C .
$$

\subsection{Discrete Fourier transform}

The discrete Fourier transform of a signal with length $N$ is ${ }^{22}$

$$
E(\omega)=\sum_{k=0}^{N-1} E(k) \exp (-j \omega k \tau) .
$$

If $E(\omega)=E_{\mathrm{r}}(\omega)+j E_{\mathrm{i}}(\omega)$, where $E_{\mathrm{r}}(\omega)$ and $E_{\mathrm{i}}(\omega)$ are real, then

$$
\begin{aligned}
& E_{\mathrm{r}}(\omega)=\sum_{k=0}^{N-1} E(k) \cos (\omega k \tau), \\
& E_{\mathrm{i}}(\omega)=-\sum_{k=0}^{N-1} E(k) \sin (\omega k \tau) .
\end{aligned}
$$

Assuming that the amplitude at each time sample is statistically independent from the amplitude at other time samples, the variances of the real and imaginary parts of the spectrum are, respectively, ${ }^{23}$

$$
\begin{aligned}
& \sigma_{E_{\mathrm{r}}}^{2}(\omega)=\sum_{k=0}^{N-1} \cos ^{2}(\omega k \tau) \sigma_{E}^{2}(k), \\
& \sigma_{E_{\mathrm{i}}}^{2}(\omega)=\sum_{k=0}^{N-1} \sin ^{2}(\omega k \tau) \sigma_{E}^{2}(k) .
\end{aligned}
$$


Since the real and imaginary parts of the spectrum use the same set of input, their covariance is then ${ }^{23}$

$$
\begin{aligned}
\sigma_{E_{\mathrm{r}} E_{\mathrm{i}}}^{2}(\omega) & =-\sum_{k=0}^{N-1} \sin (\omega k \tau) \cos (\omega k \tau) \sigma_{E}^{2}(k) \\
& =-\frac{1}{2} \sum_{k=0}^{N-1} \sin (2 \omega k \tau) \sigma_{E}^{2}(k) .
\end{aligned}
$$

\subsection{Magnitude and phase calculation}

The magnitude and phase of the signal, determined from the real and imaginary parts of the complex spectrum, are

$$
\begin{aligned}
& |E(\omega)|=\sqrt{E_{\mathrm{r}}(\omega)^{2}+E_{\mathrm{i}}(\omega)^{2}}, \\
& \angle E(\omega)=\arctan \left(E_{\mathrm{i}}(\omega) / E_{\mathrm{r}}(\omega)\right) .
\end{aligned}
$$

Correspondingly, the variances of the magnitude and phase are

$$
\begin{aligned}
\sigma_{|E|}^{2}(\omega) & =\frac{1}{|E(\omega)|^{2}}\left[E_{\mathrm{r}}(\omega)^{2} \sigma_{E_{\mathrm{r}}}^{2}(\omega)+E_{\mathrm{i}}^{2}(\omega) \sigma_{E_{\mathrm{i}}}^{2}(\omega)+2 E_{\mathrm{r}}(\omega) E_{\mathrm{i}}(\omega) \sigma_{E_{\mathrm{r}} E_{\mathrm{i}}}^{2}(\omega)\right] \\
\sigma_{\angle E}^{2}(\omega) & =\frac{1}{|E(\omega)|^{4}}\left[E_{\mathrm{i}}(\omega)^{2} \sigma_{E_{\mathrm{r}}}^{2}(\omega)+E_{\mathrm{r}}(\omega)^{2} \sigma_{E_{\mathrm{i}}}^{2}(\omega)-2 E_{\mathrm{r}}(\omega) E_{\mathrm{i}}(\omega) \sigma_{E_{\mathrm{r}} E_{\mathrm{i}}}^{2}(\omega)\right] .
\end{aligned}
$$

Substituting the variances and covariance of the real and imaginary parts from Equation 17 and 18 simplifies Equation 20a and 20b to, respectively,

$$
\begin{aligned}
\sigma_{|E|}^{2}(\omega) & =\frac{1}{|E(\omega)|^{2}} \sum_{k=0}^{N-1}\left[E_{\mathrm{r}}(\omega) \cos (\omega k \tau)-E_{\mathrm{i}}(\omega) \sin (\omega k \tau)\right]^{2} \sigma_{E}^{2}(k), \\
\sigma_{\angle E}^{2}(\omega) & =\frac{1}{|E(\omega)|^{4}} \sum_{k=0}^{N-1}\left[E_{\mathrm{i}}(\omega) \cos (\omega k \tau)+E_{\mathrm{r}}(\omega) \sin (\omega k \tau)\right]^{2} \sigma_{E}^{2}(k) .
\end{aligned}
$$

\subsection{Deconvolution}

The transfer function of a system is calculated by deconvolving a sample signal with a reference signal in the time domain. Equivalently, this operation in the frequency domain is given by magnitude dividing and phase subtraction, or

$$
\begin{aligned}
& |H(\omega)|=\left|E_{\text {sam }}(\omega)\right| /\left|E_{\text {ref }}(\omega)\right|, \\
& \angle H(\omega)=\angle E_{\text {sam }}(\omega)-\angle E_{\text {ref }}(\omega) .
\end{aligned}
$$

The magnitude and phase of signals are presumably treated as independent input parameters, and thereby there is no connection between the above two equations. The variances of Equation 22a and 22b are, respectively,

$$
\begin{aligned}
\sigma_{|H|}^{2}(\omega) & =\frac{1}{\left|E_{\mathrm{ref}}(\omega)\right|^{2}} \sigma_{\left|E_{\mathrm{sam}}\right|}^{2}(\omega)+\frac{\left|E_{\mathrm{sam}}(\omega)\right|^{2}}{\left|E_{\mathrm{ref}}(\omega)\right|^{4}} \sigma_{\left|E_{\mathrm{ref}}\right|}^{2}(\omega), \\
\sigma_{\angle H}^{2}(\omega) & =\sigma_{\angle E_{\mathrm{sam}}}^{2}(\omega)+\sigma_{\angle E_{\mathrm{ref}}}^{2}(\omega) .
\end{aligned}
$$

\subsection{Phase extrapolation}

After the deconvolution it is necessary to unwrap the phase of the transfer function. A phase unwrapping process is straightforward, and does not introduce any further uncertainty to the data. The unwrapped phase is determined by

$$
\phi(\omega)=\angle H(\omega)+2 \pi M(\omega)
$$


where $M$ is an integer, logically resolved from the phase at lower frequencies. Therefore, the phase variance is

$$
\sigma_{\phi}^{2}(\omega)=\sigma_{\angle H}^{2}(\omega)
$$

However, as the unwrapping starts from DC, it is prone to error due to low SNR for data at low frequencies. Thus, via the unwrapping process, these errors accumulate into the higher frequency range. A way to circumvent noisy phase unwrapping is to substitute the phase in the low frequency region by a phase extrapolated from a more reliable mid-frequency region. Typically, linear extrapolation suffices.

In the linear extrapolation process, a reliable part of phase is offset by bias value, $b$, obtained from the least square fitting method. Therefore, the corrected phase is

$$
\phi_{c}(\omega)=\phi(\omega)+b
$$

and the bias, $b$, is given by

$$
\begin{aligned}
b & =f\left(\phi_{1}, \phi_{2}, \ldots, \phi_{m}\right) \\
& =\frac{\sum \phi_{i} \sum \omega_{i}^{2}-\sum \omega_{i} \sum \omega_{i} \phi_{i}}{m\left(\sum \omega_{i}^{2}-\left(\sum \omega_{i}\right)^{2} / m\right)},
\end{aligned}
$$

where the summation is carried out over a high-SNR frequency range with $m$ sampling points.

From Equation 26, the variance of the corrected phase is

$$
\sigma_{\phi_{c}}^{2}(\omega)=\sigma_{\phi}^{2}(\omega)+\sigma_{b}^{2}+2 \sigma_{\phi b}^{2}(\omega) .
$$

In order to find variance, $\sigma_{b}^{2}(\omega)$, and covariance, $\sigma_{\phi b}^{2}(\omega)$, of $b$, Equation 27 is rearranged to

$$
\begin{aligned}
b & =Q \sum_{i=1}^{m} \phi_{i}-R \sum_{i=1}^{m} \omega_{i} \phi_{i} \\
& =\sum_{i=1}^{m} \phi_{i}\left(Q-R \omega_{i}\right)
\end{aligned}
$$

where

$$
\begin{aligned}
Q & =\frac{\sum_{i} \omega_{i}^{2}}{m\left(\sum_{i} \omega_{i}^{2}-\left(\sum_{i} \omega_{i}^{2}\right) / m\right)} \\
R & =\frac{\sum_{i} \omega_{i}}{m\left(\sum_{i} \omega_{i}^{2}-\left(\sum_{i} \omega_{i}^{2}\right) / m\right)} .
\end{aligned}
$$

Hence, the variance of $b$ is

$$
\sigma_{b}^{2}=\sum_{i=1}^{m} \sigma_{\phi_{i}}^{2}\left(Q-R \omega_{i}\right)^{2}
$$

and the covariance is

$$
\sigma_{\phi b}^{2}(\omega)=\sigma_{\phi}^{2}(\omega)(Q-R \omega)
$$

Notice that the bias variance is inversely proportional to the square of the number of sampling points. 


\subsection{Thickness measurement and sample alignment}

According to Figure 4, the propagation distance inside a sample, $d$, is a function of the sample thickness, $l$, and the refraction angle, $\theta_{t}$, or

$$
d=\frac{l}{\cos \theta_{t}}
$$

From Equation 33 the propagation distance variance, $\sigma_{d}^{2}$, is given by

$$
\sigma_{d}^{2}=\left(\frac{1}{\cos \theta_{t}}\right)^{2} \sigma_{l}^{2}+\left(\frac{l \sin \theta_{t}}{\cos ^{2} \theta_{t}}\right)^{2} \sigma_{\theta_{t}}^{2}
$$

The thickness variance, $\sigma_{l}^{2}$, is due to the measurement uncertainty, and the angle variance, $\sigma_{\theta_{t}}^{2}$, is due to the alignment uncertainty.

According to Snell's law, the refraction angle is derived from the incident angle, $\theta_{i}$, or

$$
\theta_{t}=\arcsin \left(\frac{n_{0} \sin \theta_{i}}{n}\right)
$$

Assuming the index of refraction of sample, $n$, and of air, $n_{0}$, are fixed over measurements, the variance in the refraction angle is therefore

$$
\sigma_{\theta_{t}}^{2}=\frac{n_{0}^{2} \cos ^{2} \theta_{i}}{n^{2}-n_{0}^{2} \sin ^{2} \theta_{i}} \sigma_{\theta_{i}}^{2}
$$

where $\sigma_{\theta_{i}}^{2}$ is the variance in the incident angle.

In fact, when the T-ray incident angle is not zero, the transfer function of the sample becomes complicated. More precisely, overly tilting of the sample will result in a complex propagation geometry, a deviated beam direction, and a lower T-ray energy focused onto a detector. In order to alleviate these complications, the incident angle is assumed to be normal to the surface, so that a simple transfer function can be adopted, and the unfocused energy can be discarded.

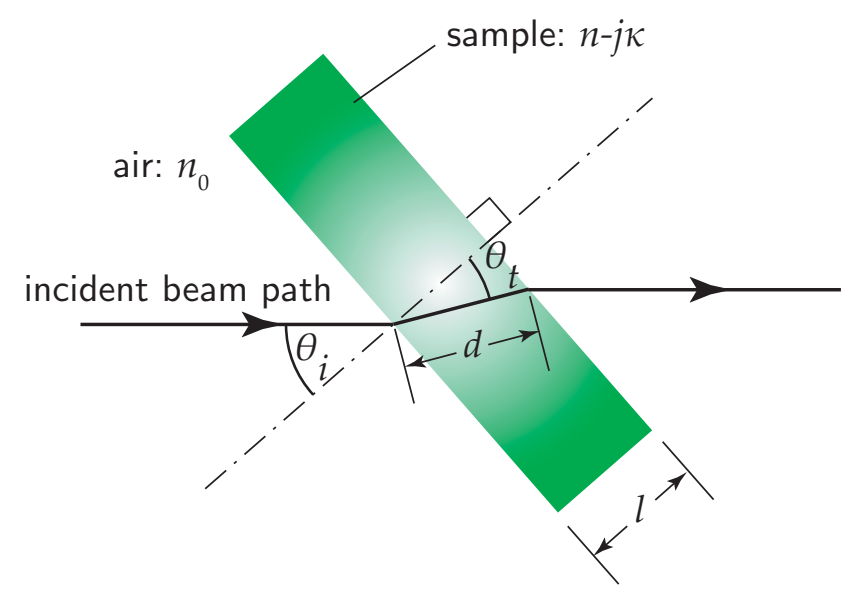

Figure 4. Tilted sample in a T-ray beam path. The T-ray path inside the sample, $d$, is longer than the sample thickness, $l$. The relation between the incident angle and refraction angle is $n \sin \theta_{t}=n_{0} \sin \theta_{i}$. This exaggerated figure illustrates a small deviation from the normal, which might occur due to sample alignment uncertainty. 


\subsection{Parameter extraction}

Once the transfer function, $H(\omega)$, of a sample is resolved by deconvolution and phase unwrapping, the optical constants of a sample are readily extractable. The optical constants comprise the index of refraction, $n(\omega)$, and the extinction coefficient, $\kappa(\omega)$, grouped together in the complex index of refraction, $\hat{n}(\omega)=n(\omega)-j \kappa(\omega)$. These quantities are frequency-dependent, and are related to the transfer function of a material slab by

$$
H(\omega)=\tau \tau^{\prime} \cdot \exp \left\{-\kappa(\omega) \frac{\omega d}{c}\right\} \cdot \exp \left\{-j\left[n(\omega)-n_{0}\right] \frac{\omega d}{c}\right\}
$$

where $\tau$ and $\tau^{\prime}$ are the transmission coefficients, which are dependent on the complex index of refraction and the incident beam polarization. However, assuming a small deviation of the angle of incident and a negligible extinction coefficient, the transmission coefficients can be approximated to

$$
\tau \tau^{\prime}=\frac{4 n(\omega) n_{0}}{\left[n(\omega)+n_{0}\right]^{2}} .
$$

The optical constants can be deduced from Equation 37 as

$$
\begin{aligned}
n(\omega) & =n_{0}-\frac{c}{\omega d} \phi_{c}(\omega), \\
\kappa(\omega) & =\frac{c}{\omega d}\left\{\ln \left[\frac{4 n(\omega) n_{0}}{\left(n(\omega)+n_{0}\right)^{2}}\right]-\ln |H(\omega)|\right\} .
\end{aligned}
$$

Hence, the absorption coefficient is

$$
\begin{aligned}
\alpha(\omega) & =\frac{2 \omega}{c} \kappa(\omega) \\
& =\frac{2}{d}\left\{\ln \left[\frac{4 n(\omega) n_{0}}{\left(n(\omega)+n_{0}\right)^{2}}\right]-\ln |H(\omega)|\right\} .
\end{aligned}
$$

From Equation 39a, the sample index uncertainty is determined by the sample thickness variance $\sigma_{d}^{2}$, the phase variance $\sigma_{\phi_{c}}^{2}(\omega)$, and the air index variance $\sigma_{n_{0}}^{2}$, or

$$
\sigma_{n}^{2}(\omega)=\left[\frac{c}{\omega d^{2}} \phi_{c}(\omega)\right]^{2} \sigma_{d}^{2}+\left[\frac{c}{\omega d}\right]^{2} \sigma_{\phi_{c}}^{2}(\omega)+\sigma_{n_{0}}^{2} .
$$

The extinction or absorption coefficient uncertainty is determined by the thickness variance $\sigma_{d}^{2}$, the amplitude variance $\sigma_{|H(\omega)|}^{2}$, the sample-index variance $\sigma_{n(\omega)}^{2}$, and the air-index variance $\sigma_{n_{0}}^{2}$. The analysis shows that

$$
\sigma_{\kappa}^{2}(\omega)=\left[\frac{\kappa(\omega)}{d}\right]^{2} \sigma_{d}^{2}+\left[\frac{c}{\omega d|H(\omega)|}\right]^{2} \sigma_{|H|}^{2}(\omega)+\left[\frac{c}{\omega d}\left(\frac{n(\omega)-n_{0}}{n(\omega)+n_{0}}\right)\right]^{2}\left[\frac{\sigma_{n}^{2}(\omega)}{n(\omega)^{2}}+\frac{\sigma_{n_{0}}^{2}}{n_{0}^{2}}\right],
$$

and, correspondingly,

$$
\begin{aligned}
\sigma_{\alpha}^{2}(\omega) & =\left(\frac{2 \omega}{c}\right)^{2} \sigma_{\kappa}^{2}(\omega) \\
& =\left[\frac{\alpha(\omega)}{d}\right]^{2} \sigma_{d}^{2}+\left[\frac{2}{d|H(\omega)|}\right]^{2} \sigma_{|H|}^{2}(\omega)+\left[\frac{2}{d}\left(\frac{n(\omega)-n_{0}}{n(\omega)+n_{0}}\right)\right]^{2}\left[\frac{\sigma_{n}^{2}(\omega)}{n(\omega)^{2}}+\frac{\sigma_{n_{0}}^{2}}{n_{0}^{2}}\right] .
\end{aligned}
$$

\section{IMPACT OF INDIVIDUAL SOURCE OF UNCERTAINTY}

In order to analyze the impact of each source of uncertainty on the overall optical constants' uncertainty, each uncertainty source is considered independently, based on the mathematical derivation given in the previous section. Subsections below present the analytical models representing the relation between a source uncertainty and the output uncertainty. Some relations are accompanied with simulations to give better insight. Note that the simulation results are presented as the standard deviation, $\sigma$, rather than the variance, $\sigma^{2}$, since the standard deviation has the same dimension as its corresponding parameter, which is more intuitive. 


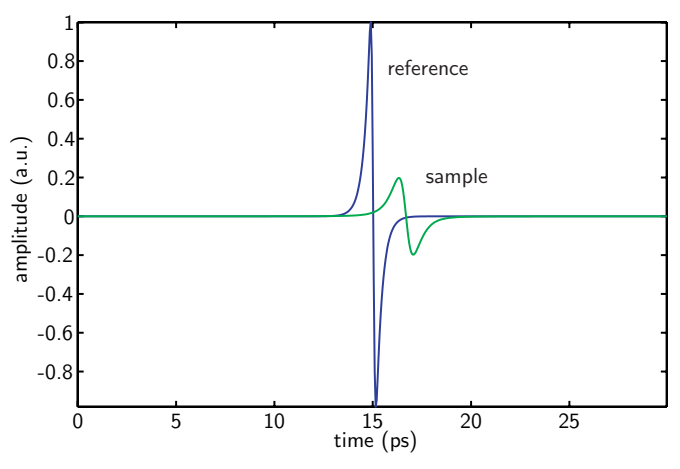

(a) Simulated T-ray signals

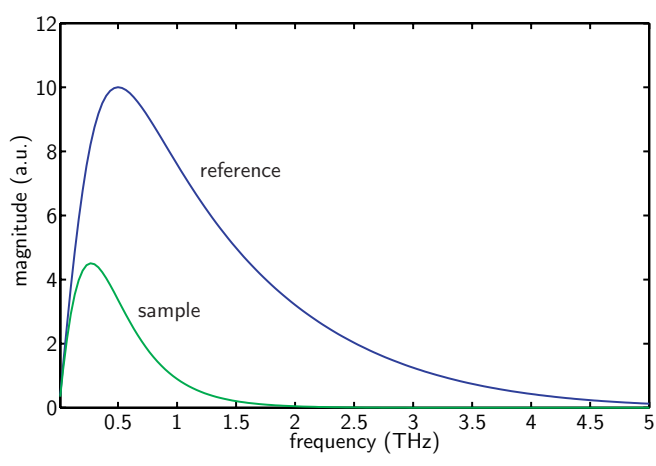

(b) Simulated T-ray spectra

Figure 5. Simulated T-ray signals and spectra for reference and sample.

\subsection{Simulation setup}

Figure 5 shows T-ray reference and sample signals simulated according to the PCA analytical model given by Duvillaret et al.. ${ }^{11}$ The reference pulse has the FWHM of approximately 0.5 ps, giving the frequency span from $0.1 \mathrm{THz}$ to $4.0 \mathrm{THz}$. The sample signal is calculated for the case that the experimental parameters are as follows: $n-j \kappa=1.5-0.1 j, l=1 \mathrm{~mm}, \theta_{i}=0^{\circ}$, and $n_{0}=1$. Note that variational ranges have been chosen arbitrarily, and might not reflect the reality.

\subsection{Impact from delay-line uncertainty}

The variances in the optical constants affected by the delay-line positioning variance, $\sigma_{x}^{2}$, are (details of derivation are elaborated in Appendix A)

$$
\begin{aligned}
\sigma_{n, x}^{2}(\omega)= & {\left[\frac{c}{\omega d}\right]^{2}\left\{\sigma_{b}^{2}+2 \sigma_{\phi b}(\omega)^{2}\right.} \\
& +\frac{1}{\left|E_{\mathrm{sam}}(\omega)\right|^{4}} \sum_{k=0}^{N-1}\left[E_{\mathrm{sam}, \mathrm{i}}(\omega) \cos (\omega k \tau)+E_{\mathrm{sam}, \mathrm{r}}(\omega) \sin (\omega k \tau)\right]^{2}\left[\frac{2}{c} \cdot \frac{\partial E_{\mathrm{sam}}(k \tau)}{\tau \partial k}\right]^{2} \sigma_{x}^{2} \\
& \left.+\frac{1}{\left|E_{\mathrm{ref}}(\omega)\right|^{4}} \sum_{k=0}^{N-1}\left[E_{\mathrm{ref}, \mathrm{i}}(\omega) \cos (\omega k \tau)+E_{\mathrm{ref}, \mathrm{r}}(\omega) \sin (\omega k \tau)\right]^{2}\left[\frac{2}{c} \cdot \frac{\partial E_{\mathrm{ref}}(k \tau)}{\tau \partial k}\right]^{2} \sigma_{x}^{2}\right\}, \\
\sigma_{\kappa, x}^{2}(\omega)= & {\left[\frac{c}{\omega d}\right]^{2}\left\{\frac{1}{\left|E_{\mathrm{sam}}(\omega)\right|^{4}} \sum_{k=0}^{N-1}\left[E_{\mathrm{sam}, \mathrm{r}}(\omega) \cos (\omega k \tau)-E_{\mathrm{sam}, \mathrm{i}}(\omega) \sin (\omega k \tau)\right]^{2}\left[\frac{2}{c} \cdot \frac{\partial E_{\mathrm{sam}}(k \tau)}{\tau \partial k}\right]^{2} \sigma_{x}^{2}\right.} \\
& +\frac{1}{\left|E_{\mathrm{ref}}(\omega)\right|^{4}} \sum_{k=0}^{N-1}\left[E_{\mathrm{ref}, \mathrm{r}}(\omega) \cos (\omega k \tau)-E_{\mathrm{ref}, \mathrm{i}}(\omega) \sin (\omega k \tau)\right]^{2}\left[\frac{2}{c} \cdot \frac{\partial E_{\mathrm{ref}}(k \tau)}{\tau \partial k}\right]^{2} \sigma_{x}^{2} \\
& \left.+\left(\frac{n(\omega)-n_{0}}{n(\omega)+n_{0}}\right)^{2} \frac{\sigma_{n, x}^{2}(\omega)}{n(\omega)^{2}}\right\} .
\end{aligned}
$$

Figures 6 illustrates the effects of delay-line positioning uncertainty. The optical constants' uncertainties change linearly and proportionally with the positioning uncertainty, and are lowest near the peak frequency.

\subsection{Impact from noise}

The variances of the optical constants affected by the noise, $A E^{2}(k \tau)+B|E(k \tau)|+C$, are (details of derivation are elaborated in Appendix A)

$\sigma_{n, E}^{2}(\omega)=\left[\frac{c}{\omega d}\right]^{2}\left\{\sigma_{b}^{2}+2 \sigma_{\phi b}(\omega)^{2}\right.$ 


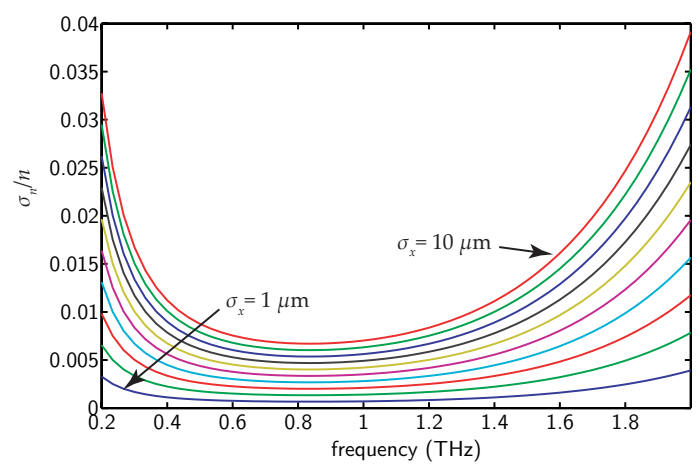

(a) Normalized standard deviation of $n$

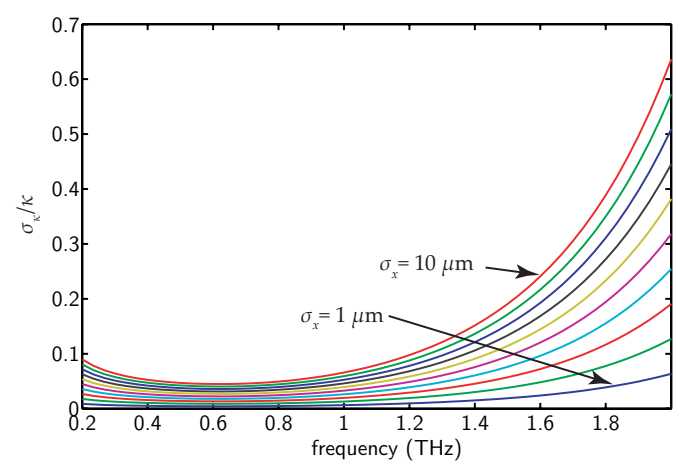

(b) Normalized standard deviation of $\kappa$

Figure 6. Standard deviation of optical constants affected by delay-line uncertainty. The standard deviation of delay line position runs from $1 \mu \mathrm{m}$ to $10 \mu \mathrm{m}$ with $1 \mu \mathrm{m}$ increment.

$$
\begin{aligned}
& +\frac{1}{\left|E_{\mathrm{sam}}(\omega)\right|^{4}} \sum_{k=0}^{N-1}\left[E_{\mathrm{sam}, \mathrm{i}}(\omega) \cos (\omega k \tau)+E_{\mathrm{sam}, \mathrm{r}}(\omega) \sin (\omega k \tau)\right]^{2}\left[A E_{\mathrm{sam}}^{2}(k \tau)+B E_{\mathrm{sam}}(k \tau)+C\right] \\
& \left.+\frac{1}{\left|E_{\mathrm{ref}}(\omega)\right|^{4}} \sum_{k=0}^{N-1}\left[E_{\mathrm{ref}, \mathrm{i}}(\omega) \cos (\omega k \tau)+E_{\mathrm{ref}, \mathrm{r}}(\omega) \sin (\omega k \tau)\right]^{2}\left[A E_{\mathrm{ref}}^{2}(k \tau)+B E_{\mathrm{ref}}(k \tau)+C\right]\right\} . \quad(45 \mathrm{a}) \\
\sigma_{\kappa, E}^{2}(\omega)= & {\left[\frac{c}{\omega d}\right]^{2}\left\{\frac{1}{\left|E_{\mathrm{sam}}(\omega)\right|^{4}} \sum_{k=0}^{N-1}\left[E_{\mathrm{sam}, \mathrm{r}}(\omega) \cos (\omega k \tau)-E_{\mathrm{sam}, \mathrm{i}}(\omega) \sin (\omega k \tau)\right]^{2}\left[A E_{\mathrm{sam}}^{2}(k \tau)+B E_{\mathrm{sam}}(k \tau)+C\right]\right.} \\
& +\frac{1}{\left|E_{\mathrm{ref}}(\omega)\right|^{4}} \sum_{k=0}^{N-1}\left[E_{\mathrm{ref}, \mathrm{r}}(\omega) \cos (\omega k \tau)-E_{\mathrm{ref}, \mathrm{i}}(\omega) \sin (\omega k \tau)\right]^{2}\left[A E_{\mathrm{ref}}^{2}(k \tau)+B E_{\mathrm{ref}}(k \tau)+C\right] \\
& \left.+\left(\frac{n(\omega)-n_{0}}{n(\omega)+n_{0}}\right)^{2} \frac{\sigma_{n, E}^{2}(\omega)}{n(\omega)^{2}}\right\} .
\end{aligned}
$$

Figures 7 illustrates the effect of the inevitable electronic and optic noises on the output uncertainties. A higher order coefficient, e.g. $A$, has less impact on the variation at high frequencies, due to the amplitude dependence of noise.

\subsection{Impact from air refractive index uncertainty}

The variances of the optical constants affected by the air-index variance, $\sigma_{n_{0}}^{2}$, are

$$
\begin{aligned}
\sigma_{n, n_{0}}^{2}(\omega) & =\sigma_{n_{0}}^{2} \\
\sigma_{\kappa, n_{0}}^{2}(\omega) & =\left[\frac{c}{\omega d}\left(\frac{n(\omega)-n_{0}}{n(\omega)+n_{0}}\right)\right]^{2}\left[\frac{1}{n(\omega)^{2}}+\frac{1}{n_{0}^{2}}\right] \sigma_{n_{0}}^{2} .
\end{aligned}
$$

The variances of both optical constants varies linearly with the variance of the air refractive index. Also the variance of the extinction coefficient decreases with frequency, as appearing in Figure 8.

\subsection{Impact from sample thickness uncertainty}

The variances of the optical constants affected by the sample thickness variance, $\sigma_{l}^{2}$, are

$$
\begin{aligned}
\sigma_{n, l}^{2}(\omega) & =\left[\frac{c}{\omega l^{2}} \phi_{c}(\omega) \cos \theta_{t}\right]^{2} \sigma_{l}^{2} \\
\sigma_{\kappa, l}^{2}(\omega) & =\left[\frac{\kappa(\omega)}{l}\right]^{2} \sigma_{l}^{2}+\left[\frac{c}{\omega d n(\omega)}\left(\frac{n(\omega)-n_{0}}{n(\omega)+n_{0}}\right)\right]^{2} \sigma_{n, l}^{2}(\omega) .
\end{aligned}
$$




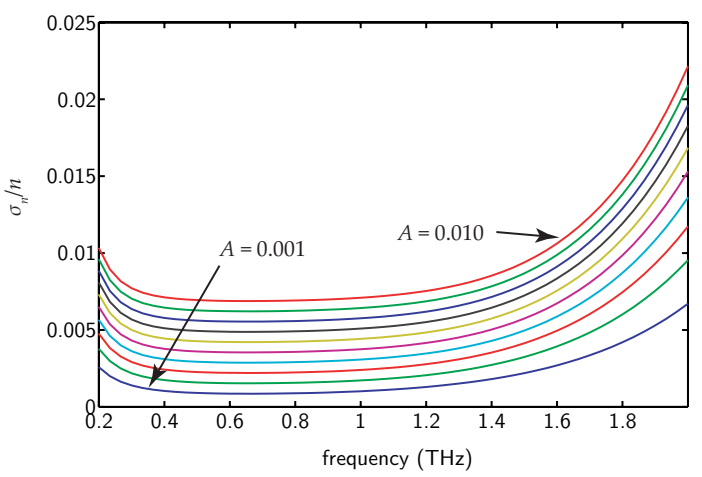

(a) Standard deviation of $n$ affected by $A E^{2}(k \tau)$

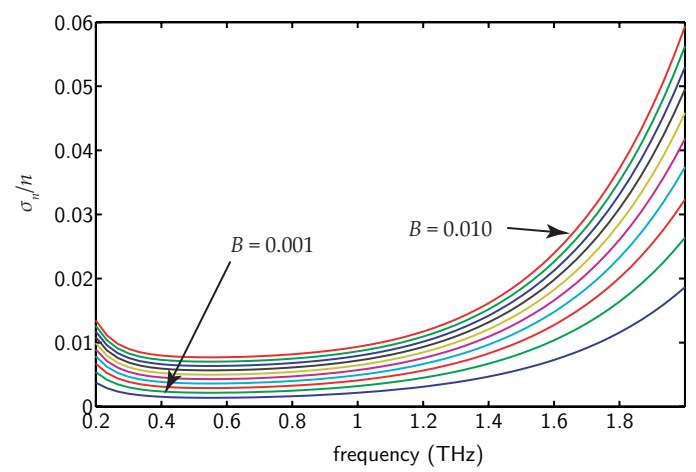

(c) Standard deviation of $n$ affected by $B|E(k \tau)|$

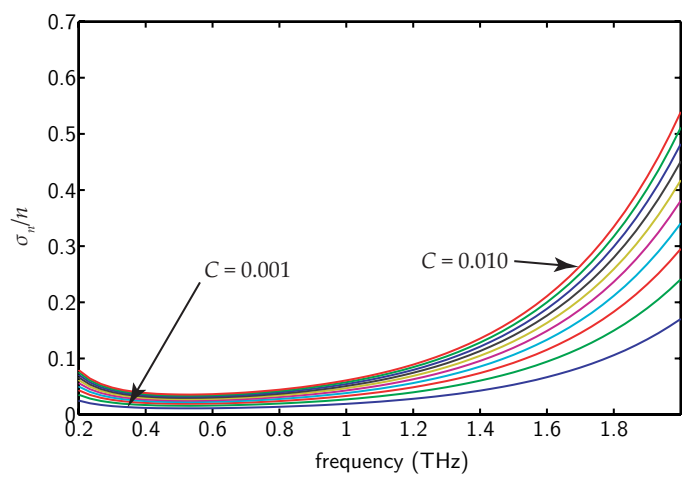

(e) Standard deviation of $n$ affected by $C$

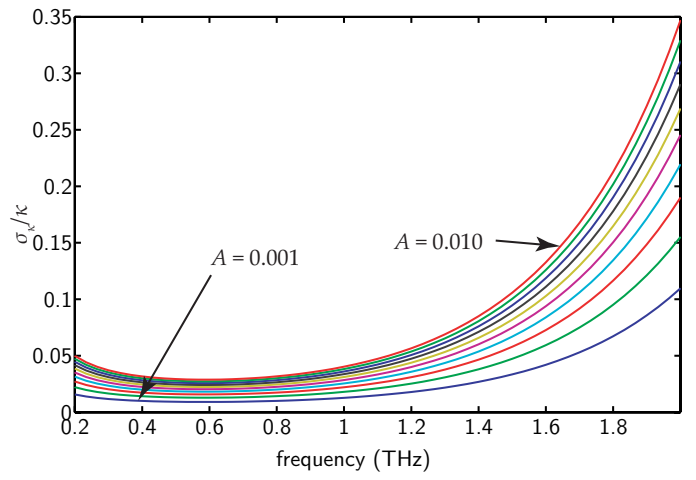

(b) Standard deviation of $\kappa$ affected by $A E^{2}(k \tau)$

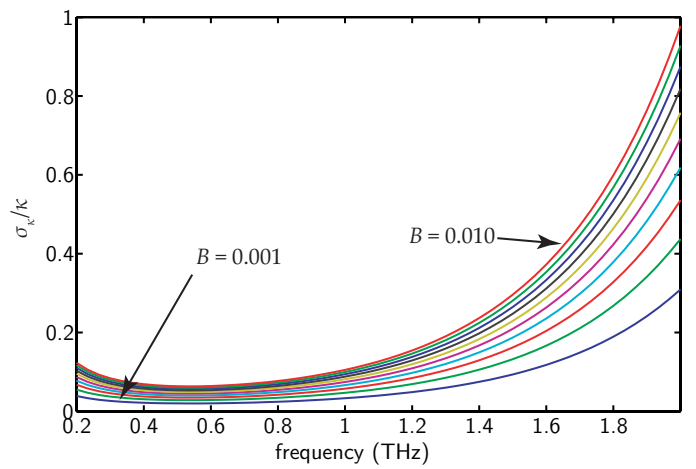

(d) Standard deviation of $\kappa$ affected by $B|E(k \tau)|$

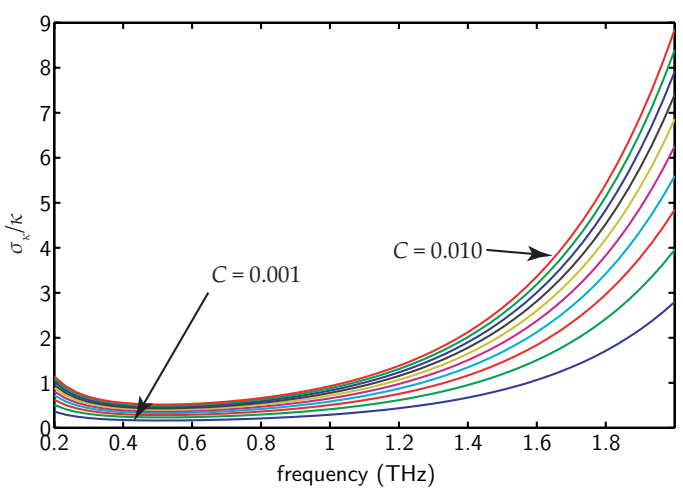

(f) Standard deviation of $\kappa$ affected by $C$

Figure 7. Standard deviation of optical constants affected by noise. The noise's amplitude coefficients, $A, B$, and $C$, run from 0.001 to 0.010 with 0.001 increment. 


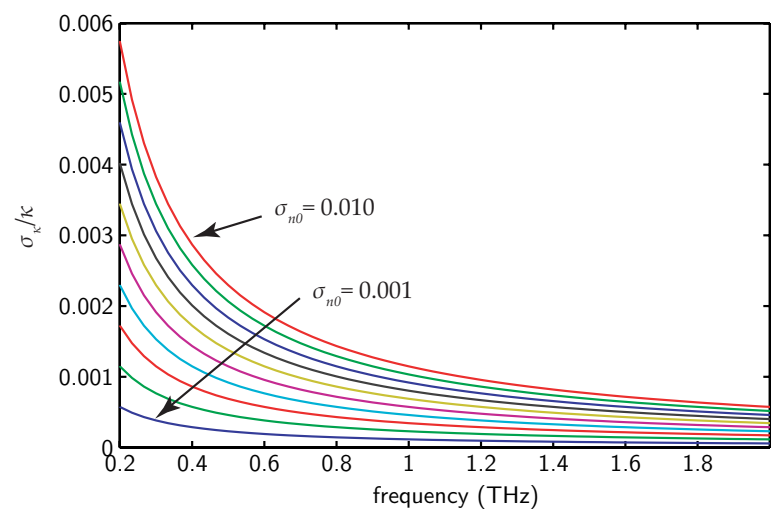

Figure 8. Normalized standard deviation of the extinction coefficient affected by the air refractive index uncertainty. The standard deviation of the air index increases from 0.001 to 0.010 with a step of 0.001 .

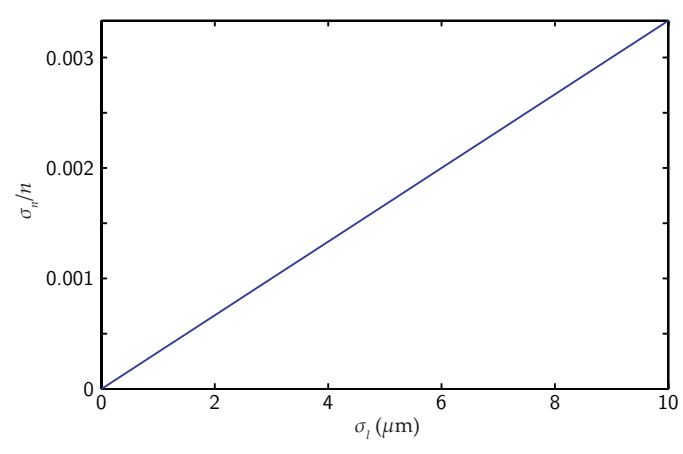

(a) Normalized standard deviation of $n$

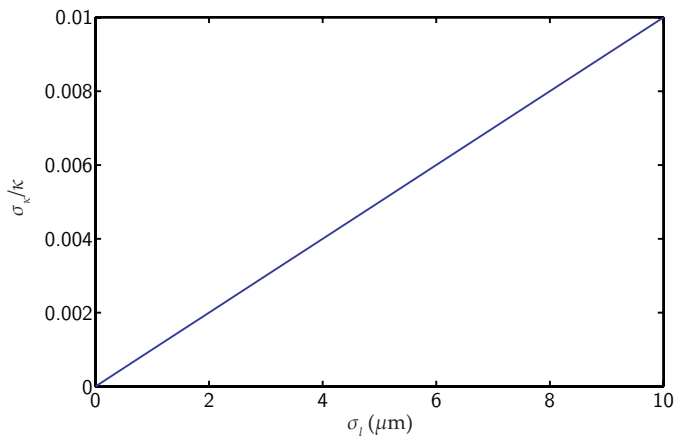

(b) Normalized standard deviation of $\kappa$

Figure 9. Standard deviation of optical constants affected by the uncertainty in sample thickness. The standard deviation of the thickness runs from $1 \mu \mathrm{m}$ to $10 \mu \mathrm{m}$ with $1 \mu \mathrm{m}$ step size.

Figures 9 illustrates the effect of uncertainty in the sample thickness on the output uncertainties. The thickness considered is in the order of a millimeter, whereas the standard deviation is around a few microns. Based on the analytical model, the uncertainties would vary with frequency, but this does not appear to be the case as found in the simulation. Given that the phase term, $\phi_{c}$, is proportional to $\omega$ (Equation 37), $\omega$ terms cancel out leaving only constants in the model. Thus, for the fixed optical constants, both $\sigma_{n, l}^{2}(\omega)$ and $\sigma_{\kappa, l}^{2}(\omega)$ vary linearly with the thickness variance but not the frequency.

\subsection{Impact from sample alignment uncertainty}

The variance of the optical constants affected by the incident angle variance, $\sigma_{\theta_{i}}^{2}$, are

$$
\begin{aligned}
\sigma_{n, \theta}^{2}(\omega) & =\frac{1}{n^{2}-n_{0}^{2} \sin ^{2} \theta_{i}}\left[\frac{n_{0} c}{\omega d} \phi_{c}(\omega) \cos \theta_{i} \tan \theta_{t}\right]^{2} \sigma_{\theta_{i}}^{2}, \\
\sigma_{\kappa, \theta}^{2}(\omega) & =\frac{\left[n_{0} \kappa(\omega) \cos \theta_{i} \tan \theta_{t}\right]^{2}}{n^{2}-n_{0}^{2} \sin ^{2} \theta_{i}} \sigma_{\theta_{i}}^{2}+\left[\frac{c}{\omega d n(\omega)}\left(\frac{n(\omega)-n_{0}}{n(\omega)+n_{0}}\right)\right]^{2} \sigma_{n, \theta}^{2}(\omega) .
\end{aligned}
$$

As the incident angle (and thus the refraction angle) is set to zero, in both equations above, $\tan \theta_{t}$ yields zero. This does not imply that the variances in the optical constants are independent of the variance of the angle of incidence. Further study indicates that the above relation cannot be dealt with the first-order approximation of variance, which was derived in Section 4.1. A higher-order relation is sought in the future work. 


\subsection{Impact from two uncertainty sources or more}

The total variance in refractive index and extinction coefficient is given by

$$
\begin{aligned}
& \sigma_{n}^{2}(\omega)=\sigma_{n, x}^{2}(\omega)+\sigma_{n, E}^{2}(\omega)+\sigma_{n, l}^{2}(\omega)+\sigma_{n, \theta}^{2}(\omega)+\sigma_{n_{0}}^{2}(\omega), \\
& \sigma_{\kappa}^{2}(\omega)=\sigma_{\kappa, x}^{2}(\omega)+\sigma_{\kappa, E}^{2}(\omega)+\sigma_{\kappa, l}^{2}(\omega)+\sigma_{\kappa, \theta}^{2}(\omega)+\sigma_{\kappa, n_{0}}^{2}(\omega),
\end{aligned}
$$

respectively - where simple addition in quadrature is carried out as the sources of uncertainty are uncorrelated.

\section{DISCUSSION AND CONCLUSION}

This paper has presented an uncertainty analysis of THz-TDS. The various sources of uncertainty, appearing in THz-TDS and throughout the parameter extraction process, are identified and modeled mathematically. The propagation of uncertainties from these sources to the complex index of refraction is estimated. The relation between each source and the optical constants' uncertainties is considered in detail, and accompanied by the simulation results.

The proposed model is applicable to either PCA or EO generation and detection systems. It should, however, be noted that the model is first-order approximated, which is valid in case where sources of uncertainty have variation limited to a small vicinity.

Future work, which we are preparing, will endeavor to investigate each uncertainty source in depth and provide both theoretical and practical suggestions for system optimization. A higher order analysis of uncertainty is demanded for high accuracy uncertainty estimation. Future experiments to substantiate the developed model will also be carried out.

\section{APPENDIX A. DERIVATION OF OPTICAL CONSTANTS' VARIANCES AFFECTED BY AMPLITUDE UNCERTAINTY}

The direct relation between the variances in the optical constants, $\sigma_{n}^{2}(\omega)$ and $\sigma_{\kappa}^{2}(\omega)$, and the amplitude variance, $\sigma_{E}^{2}(k)$, is derived in this section based on the analysis given in Section 5.

According to Equation 21a the amplitude variances of the sample and reference spectra are given by, respectively,

$$
\begin{aligned}
\sigma_{\left|E_{\mathrm{sam}}\right|}^{2}(\omega) & =\frac{1}{\left|E_{\mathrm{sam}}(\omega)\right|^{2}} \sum_{k=0}^{N-1}\left[E_{\mathrm{sam}, \mathrm{r}}(\omega) \cos (\omega k \tau)-E_{\mathrm{sam}, \mathrm{i}}(\omega) \sin (\omega k \tau)\right]^{2} \sigma_{E_{\mathrm{sam}}}^{2}(k), \\
\sigma_{\left|E_{\mathrm{ref}}\right|}^{2}(\omega) & =\frac{1}{\left|E_{\mathrm{ref}}(\omega)\right|^{2}} \sum_{k=0}^{N-1}\left[E_{\mathrm{ref}, \mathrm{r}}(\omega) \cos (\omega k \tau)-E_{\mathrm{ref}, \mathrm{i}}(\omega) \sin (\omega k \tau)\right]^{2} \sigma_{E_{\mathrm{ref}}}^{2}(k),
\end{aligned}
$$

and according to Equation $21 \mathrm{~b}$ the phase variances of the sample and reference spectra are given by, respectively,

$$
\begin{aligned}
\sigma_{\angle E_{\mathrm{sam}}}^{2}(\omega) & =\frac{1}{\left|E_{\mathrm{sam}}(\omega)\right|^{4}} \sum_{k=0}^{N-1}\left[E_{\mathrm{sam}, \mathrm{i}}(\omega) \cos (\omega k \tau)+E_{\mathrm{sam}, \mathrm{r}}(\omega) \sin (\omega k \tau)\right]^{2} \sigma_{E_{\mathrm{sam}}}^{2}(k), \\
\sigma_{\angle E_{\mathrm{ref}}}^{2}(\omega) & =\frac{1}{\left|E_{\mathrm{ref}}(\omega)\right|^{4}} \sum_{k=0}^{N-1}\left[E_{\mathrm{ref}, \mathrm{i}}(\omega) \cos (\omega k \tau)+E_{\mathrm{ref}, \mathrm{r}}(\omega) \sin (\omega k \tau)\right]^{2} \sigma_{E_{\mathrm{ref}}}^{2}(k) .
\end{aligned}
$$

Substitute the above four equations into Equation 23a and 23b gives

$$
\begin{aligned}
\sigma_{|H|}^{2}(\omega)= & \frac{1}{\left|E_{\mathrm{ref}}(\omega)\right|^{2}\left|E_{\mathrm{sam}}(\omega)\right|^{2}} \sum_{k=0}^{N-1}\left[E_{\mathrm{sam}, \mathrm{r}}(\omega) \cos (\omega k \tau)-E_{\mathrm{sam}, \mathrm{i}}(\omega) \sin (\omega k \tau)\right]^{2} \sigma_{E_{\mathrm{sam}}}^{2}(k) \\
& +\frac{\left|E_{\mathrm{sam}}(\omega)\right|^{2}}{\left|E_{\mathrm{ref}}(\omega)\right|^{6}} \sum_{k=0}^{N-1}\left[E_{\mathrm{ref}, \mathrm{r}}(\omega) \cos (\omega k \tau)-E_{\mathrm{ref}, \mathrm{i}}(\omega) \sin (\omega k \tau)\right]^{2} \sigma_{E_{\mathrm{ref}}}^{2}(k),
\end{aligned}
$$


and

$$
\begin{aligned}
\sigma_{\angle H}^{2}(\omega)= & \frac{1}{\left|E_{\mathrm{sam}}(\omega)\right|^{4}} \sum_{k=0}^{N-1}\left[E_{\mathrm{sam}, \mathrm{i}}(\omega) \cos (\omega k \tau)+E_{\mathrm{sam}, \mathrm{r}}(\omega) \sin (\omega k \tau)\right]^{2} \sigma_{E_{\mathrm{sam}}}^{2}(k) \\
& +\frac{1}{\left|E_{\mathrm{ref}}(\omega)\right|^{4}} \sum_{k=0}^{N-1}\left[E_{\mathrm{ref}, \mathrm{i}}(\omega) \cos (\omega k \tau)+E_{\mathrm{ref}, \mathrm{r}}(\omega) \sin (\omega k \tau)\right]^{2} \sigma_{E_{\mathrm{ref}}}^{2}(k),
\end{aligned}
$$

respectively.

Equation 53 are then combined with Equations 25, 28, and 41 to derive

$$
\begin{aligned}
\sigma_{n}^{2}(\omega)= & {\left[\frac{c}{\omega d}\right]^{2}\left\{\sigma_{b}^{2}+2 \sigma_{\phi b}(\omega)^{2}\right.} \\
& +\frac{1}{\left|E_{\mathrm{sam}}(\omega)\right|^{4}} \sum_{k=0}^{N-1}\left[E_{\mathrm{sam}, \mathrm{i}}(\omega) \cos (\omega k \tau)+E_{\mathrm{sam}, \mathrm{r}}(\omega) \sin (\omega k \tau)\right]^{2} \sigma_{E_{\mathrm{sam}}}^{2}(k) \\
& \left.+\frac{1}{\left|E_{\mathrm{ref}}(\omega)\right|^{4}} \sum_{k=0}^{N-1}\left[E_{\mathrm{ref}, \mathrm{i}}(\omega) \cos (\omega k \tau)+E_{\mathrm{ref}, \mathrm{r}}(\omega) \sin (\omega k \tau)\right]^{2} \sigma_{E_{\mathrm{ref}}}^{2}(k)\right\} .
\end{aligned}
$$

Equation 52 is substituted into Equation 42 to derive

$$
\begin{aligned}
\sigma_{\kappa}^{2}(\omega)= & {\left[\frac{c}{\omega d}\right]^{2}\left\{\frac{1}{\left|E_{\mathrm{sam}}(\omega)\right|^{4}} \sum_{k=0}^{N-1}\left[E_{\mathrm{sam}, \mathrm{r}}(\omega) \cos (\omega k \tau)-E_{\mathrm{sam}, \mathrm{i}}(\omega) \sin (\omega k \tau)\right]^{2} \sigma_{E_{\mathrm{sam}}}^{2}(k)\right.} \\
& +\frac{1}{\left|E_{\mathrm{ref}}(\omega)\right|^{4}} \sum_{k=0}^{N-1}\left[E_{\mathrm{ref}, \mathrm{r}}(\omega) \cos (\omega k \tau)-E_{\mathrm{ref}, \mathrm{i}}(\omega) \sin (\omega k \tau)\right]^{2} \sigma_{E_{\mathrm{ref}}}^{2}(k) \\
& \left.+\left(\frac{n(\omega)-n_{0}}{n(\omega)+n_{0}}\right)^{2} \frac{\sigma_{n}^{2}(\omega)}{n(\omega)^{2}}\right\} .
\end{aligned}
$$

In Equations 54 and 55, in case that the variance in the delay-line positioning is considered, $\sigma_{E_{\text {ref }}}^{2}(k)$ and $\sigma_{E_{\text {sam }}}^{2}(k)$ can be substituted by

$$
\begin{aligned}
\sigma_{E_{\mathrm{ref}}}^{2}(k) & =\left[\frac{2}{c} \cdot \frac{\partial E_{\mathrm{ref}}(k \tau)}{\tau \partial k}\right]^{2} \sigma_{x}^{2} \quad \text { and } \\
\sigma_{E_{\mathrm{sam}}}^{2}(k) & =\left[\frac{2}{c} \cdot \frac{\partial E_{\mathrm{sam}}(k \tau)}{\tau \partial k}\right]^{2} \sigma_{x}^{2},
\end{aligned}
$$

respectively, and in case that the system's noise is considered,

$$
\begin{aligned}
\sigma_{E_{\text {ref }}}^{2}(k) & =A E_{\text {ref }}^{2}(k \tau)+B\left|E_{\mathrm{ref}}(k \tau)\right|+C \quad \text { and } \\
\sigma_{E_{\text {sam }}}^{2}(k) & =A E_{\mathrm{sam}}^{2}(k \tau)+B\left|E_{\mathrm{sam}}(k \tau)\right|+C,
\end{aligned}
$$

respectively.

\section{REFERENCES}

1. P. Smith, D. H. Auston, and M. C. Nuss, "Subpicosecond photoconducting dipole antennas," IEEE Journal of Quantum Electronics 24(2), pp. 255-260, 1988.

2. L. Xu, X.-C. Zhang, and D. H. Auston, "Terahertz beam generation by femtosecond optical pulses in electro-optic materials," Applied Physics Letters 61(15), pp. 1784-1786, 1992. 
3. L. Duvillaret, F. Garet, and J.-L. Coutaz, "A reliable method for extraction of material parameters in terahertz time-domain spectroscopy," IEEE Journal of Selected Topics in Quantum Electronics 2(3), pp. 739$746,1996$.

4. W. Withayachumnankul, B. Ferguson, T. Rainsford, S. P. Mickan, and D. Abbott, "Simple material parameter estimation via terahertz time-domain spectroscopy," IEE Electronics Letters 41(14), pp. 800-801, 2005.

5. H. A. Haus and A. Mecozzi, "Noise of mode-locked lasers," IEEE Journal of Quantum Electronics 29(3), pp. 983-996, 1993.

6. A. Poppe, L. Xu, F. Krausz, and C. Spielmann, "Noise characterization of sub-10-fs Ti:sapphire oscillators," IEEE Journal of Selected Topics in Quantum Electronics 4(2), pp. 179-184, 1998.

7. M. van Exter, C. Fattinger, and D. Grischkowsky, "Terahertz time-domain spectroscopy of water vapor," Optics Letters 14(20), pp. 1128-1130, 1989.

8. W. Withayachumnankul, B. Ferguson, T. Rainsford, S. P. Mickan, and D. Abbott, "Direct Fabry-Pérot effect removal," Fluctuation and Noise Letters 6(2), pp. L227-L239, 2006.

9. M. van Exter and D. R. Grischkowsky, "Characterization of an optoelectronic terahertz beam system," IEEE Transactions on Microwave Theory and Techniques 38(11), pp. 1684-1691, 1990.

10. L. Duvillaret, F. Garet, and J.-L. Coutaz, "Influence of noise on the characterization of materials by terahertz time-domain spectroscopy," Journal of the Optical Society of America B: Optical Physics 17(3), pp. 452-460, 2000.

11. L. Duvillaret, F. Garet, J.-F. Roux, and J.-L. Coutaz, "Analytical modeling and optimization of terahertz time-domain spectroscopy experiments using photoswitches as antennas," IEEE Journal of Selected Topics in Quantum Electronics 7(4), pp. 615-623, 2001.

12. P. U. Jepsen, R. H. Jacobsen, and S. R. Keiding, "Generation and detection of terahertz pulses from biased semiconductor antennas," Journal of the Optical Society of America B: Optical Physics 13(11), pp. 24242436, 1996.

13. K.-S. Lee, T.-M. Lu, and X.-C. Zhang, "Tera tool," IEEE Circuits $\&$ Devices Magazine 18(6), pp. 23-28, 2002.

14. Z. Jiang, M. Li, and X.-C. Zhang, "Dielectric constant measurement of thin films by differential time-domain spectroscopy," Applied Physics Letters 76(22), pp. 3221-3223, 2000.

15. B. Ferguson and D. Abbott, "Wavelet de-noising of optical terahertz pulse imaging data," Journal of Fluctuation and Noise Letters 1(2), pp. L65-L69, 2001.

16. B. Ferguson and D. Abbott, "De-noising techniques for terahertz responses of biological samples," Microelectronics Journal 32(12), pp. 943-953, 2001.

17. I. Pupeza, R. Wilk, and M. Koch, "Highly accurate optical material parameter determination with $\mathrm{THz}$ time domain spectroscopy," Optics Express 15(7), pp. 4335-4350, 2007.

18. J. Xu, T. Yuan, S. Mickan, and X. C. Zhang, "Limit of spectral resolution in terahertz time-domain spectroscopy," Chinese Physics Letters 20(8), pp. 1266-1268, 2003.

19. S. P. Mickan, J. Xu, J. Munch, X.-C. Zhang, and D. Abbott, "The limit of spectral resolution in THz time-domain spectroscopy," in Proceedings of SPIE Photonics: Design, Technology, and Packaging, 5277, pp. 54-64, 2004.

20. P. U. Jepsen and B. M. Fischer, "Dynamic range in terahertz time-domain transmission and reflection spectroscopy," Optics Letters 30(1), pp. 29-31, 2005.

21. J. Letosa, M. García-Gracia, J. M. Forniés-Marquina, and J. M. Artacho, "Performance limits in TDR technique by Monte Carlo simulation," IEEE Transactions on Magnetics 32(3), pp. 958-961, 1996.

22. W. H. Press, S. A. Teukolsky, W. T. Vetterling, and B. P. Flannery, Numerical Recipes in C: The Art of Scientific Computing, Cambridge University Press, New York, NY, USA, 1992.

23. J. M. Forniés-Marquina, J. Letosa, M. García-Gracia, and J. M. Artacho, "Error propagation for the transformation of time domain into frequency domain," IEEE Transactions on Magnetics 33(2), pp. 1456-1459, 1997. 